\title{
Cerebral microdialysis in TBI-limitations and possibilities
}

\author{
Carl-Henrik Nordström ${ }^{1}$ (iD
}

Received: 6 September 2017 / Accepted: 19 September 2017 / Published online: 12 October 2017

(C) Springer-Verlag GmbH Austria 2017

This issue of Acta Neurochirurgica contains a systematic review of all published reports on cerebral microdialysis in patients with traumatic brain injuries (TBI) [1]. The review represents a major effort to summarize the clinical experience up to now. It summarizes all reports regarding the relationship between routinely monitored biochemical variables and patient functional outcome, neurophysiologic measures and tissue outcome. The strength of evidence obtained from published data is evaluated using the Grading of Recommendation Assessment Development and Education (GRADE) criteria [2].

The scientific value of a systematic review primarily depends on a strict selection of relevant studies. The Cochrane Handbook for Systematic Reviews describes in detail how this procedure should be conducted [3]. In this handbook the difference between a study and a report is underscored. The authors of the present review have decided not to follow these strict rules and have included all published reports (e.g., abstracts, congress reports, retrospective analyses, structured full articles). In this way, the authors intend to illustrate the whole spectrum of experiences within the field. However, as they present reports and not studies, they circumvent important demands for a systematic review. The risk that several reports represent the same study is not eliminated; many reports include a very limited number of patients, which are usually illdefined, and the scientific quality of congress reports and abstracts is usually not possible to assess. As the basic require-

Carl-Henrik Nordström

carl-henrik.nordstrom@med.lu.se

1 Odense, Denmark ments for a systematic review are not fulfilled, the strength of evidence presented will be of limited value, and some conclusions may even be misleading.

The limitations and possibilities inherent in the technique of microdialysis have been described in detail in many publications [4]. In the present context, the fact that microdialysis gives information regarding chemical variables from a very narrow zone surrounding the catheter constitutes an important limitation. In patients with TBI, energy metabolism is usually very different in different parts of the brain. Accordingly, efforts to correlate the biochemical variables obtained by microdialysis to clinical outcome are justified on the condition that the tissue monitored is representative of a relatively large part of the hemisphere. This is not the case in the majority of the reports summarized in the present review.

The routinely monitored chemical variables are related to cerebral energy metabolism (Fig. 1). These variables are analyzed bedside utilizing enzymatic techniques, and the analytical precision of these methods has been documented [4]. The precision is sufficient for clinical routine but is insufficient for the detection and interpretation of previously unknown biochemical principles or pathophysiological mechanisms. Accordingly, the interpretations should be based on previous knowledge of cerebral energy metabolism established in wellcontrolled experimental studies. The most relevant aspects have been known for decades [5].

Cerebral energy metabolism is strictly aerobic, and the variables monitored (glucose, pyruvate, lactate, glutamate, glycerol) are closely interrelated (Fig. 1). Compromised energy metabolism will (with one exception-systemic hypoglycemia), always cause a shift in the cytoplasmatic redox state that is immediately reflected in an increase of the lactate/pyruvate ratio (LPR). Accordingly, it is not justified to present and interpret the chemical variables monitored as being independent of each other. In particular, there is no reason to expect or 
Fig. 1 Schematic diagram of cerebral intermediary metabolism, with a focus on the glycolytic chain and its relation to glycerol and glycerophospholipids and to the citric acid cycle (Krebs cycle). F-1,6-DP: fructose-1,6diposphate; DHAP: dihydroxyacetone-phosphate; GA-3P: glyceraldehyde-3phosphate; G-3-P: glycerol-3phosphate; FFA: free fatty acids; $\alpha$-KG: $\alpha$-ketoglutarate. Underlined metabolites are measured bedside with enzymatic techniques

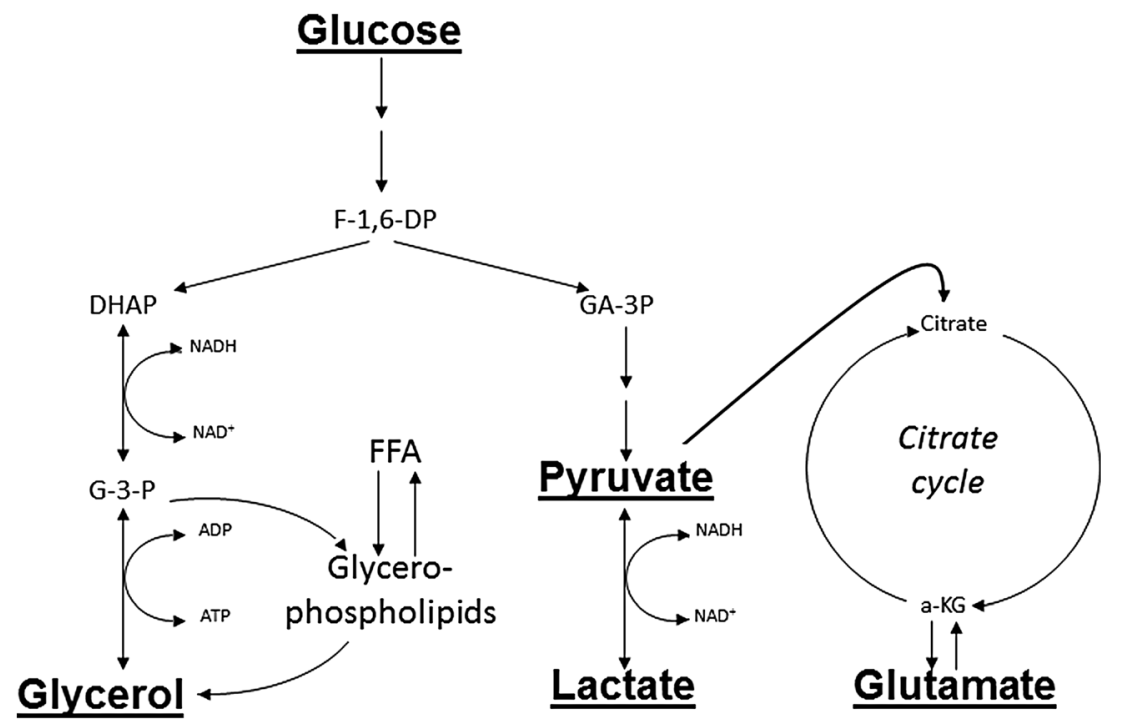

evaluate a possible correlation between each of these chemical variables and clinical outcome separately.

The section describing possible relationships between microdialysis and neurophysiologic measures illustrates a similar problem. It is unjustified to correlate individual biochemical variables to physiological measures without understanding and clarifying the underlying relationship. It should also be clarified under what conditions it is justified to correlate very local biochemical data to global physiological measures. If not, the conclusions arrived at risk being confusing or misleading. Don't we all know that high ICP/low CPP is related to compromised cerebral energy metabolism even if the relation has not been documented in some ill-defined studies in headinjured patients? Don't we all know under what circumstances local LPR and local $\mathrm{PbtO}_{2}$ may be correlated? The former variable reflects the cytoplasmatic redox state and the latter gives tissue oxygen tension. Accordingly, a correlation would be expected in ischemia but should not occur during mitochondrial dysfunction. As both conditions are common in TBI patients, it is necessary to identify and separate these two groups of patients before a possible correlation is examined. If this distinction is not performed by necessity the evaluation will result in a GRADE C (low) level of association without contributing with any information.

The third section of the review examines the relationship between microdialysis and local tissue outcome. Unfortunately only one published study was found and included. The evaluation of the relationship concludes that there is only a GRADE D (very low) level of evidence to suggest an association between LPR in the acute/ subacute phase of illness and local tissue outcome. Isn't that a misleading conclusion? Do we have any reason to doubt that severely compromised cerebral energy metabolism will result in tissue damage?
The systematic review by Zeiler et al. [1] is important as it reveals how clinical microdialysis has been used and interpreted in TBI patients up to now. A majority of the reports have been devoted to examine whether microdialysis predicts clinical outcome or whether the data obtained correlate with those obtained from other monitoring techniques. But are these aspects really of central importance during critical care? Do we really use our monitoring techniques primarily to predict clinical outcome? Do we really need various monitoring techniques that give essentially identical information?

The experiences summarized in the review underscore that future studies should have a more appropriate focus to be of real importance for neurocritical care. First, as a basis for all interpretations, it is necessary to establish the levels of the studied biochemical variables in normal human brain under physiological conditions. Presently, only two relatively small studies have been published within this field [6,7]. Second, clinical microdialysis was developed to give an early warning of deteriorating cerebral energy metabolism. Future studies should be directed towards illuminating how the information obtained may be used to change therapy and how the change of therapy affects cerebral energy state and, possibly, clinical outcome. Third, the present biochemical analysis is too laborious to become a generally established routine during neurocritical care. To achieve this goal it is necessary to develop and evaluate online analytical techniques based on a combination of microdialysis and biochemical sensors. Finally, the present very local microdialysis technique was developed to give an early warning of biochemical deterioration before it is reflected in global physiological measures or clinical symptoms. This can be achieved provided the catheter is placed within the especially vulnerable "penumbra" zones [8]. It would be of clinical importance to modify the technique to also evaluate the "global cerebral energy state." Such a 
technique might be used to predict clinical outcome in situations where this information is of particular importance (e.g., resuscitation after cardiac standstill).

\section{References}

1. Zeiler FA, Thelin E, Helmy A, Czosnyka M, Hutchinson PJ, Menon DK A (2017) Systematic review of cerebral microdialysis and outcomes in TBI: Relationships to patient functional outcome, neurophysiologic measures and tissue outcome. Acta Neurochir. https:// doi.org/10.1007/s00701-017-3338-2

2. Schünemann HJ, Oxman AD, Brozek J, Glasziou P, Jaeschke R, Vist GE, Williams JW Jr, Kunz R, Craig J, Montori VM, Bossuyt P, Guyatt GH, GRADE Working Group (2008) Grading quality of evidence and strength of recommendations for diagnostic tests and strategies. BMJ 336(7653):1106-1110
3. Higgins JPT, Green S (eds) Cochrane Handbook for Systematic Reviews of Interventions Version 5.1.0. http://handbook.cochrane. org. Accessed 25 Oct 2013

4. Nordström CH, Koskinen LO, Olivecrona M (2017) Aspects on the physiological and biochemical foundations of neurocritical care. Front Neurol 8:274

5. Siesjö BK (1978) Brain Energy Metabolism. John Wiley, Chichester

6. Reinstrup $P$, Ståhl N, Mellergård $P$, Uski T, Ungerstedt U, Nordström $\mathrm{CH}$ (2000) Intracerebral microdialysis in clinical practice. Normal values and variations during anaesthesia and neurosurgical operations. Neurosurgery 47:701-710

7. Sánchez-Guerrero A, Mur-Bonet G, Vidal-Jorge M, GándaraSabatini D, Chocrón I, Cordero E, Poca MA, Mullen K, Sahuquillo J (2017) Reappraisal of the reference levels for energy metabolites in the extracellular fluid of the human brain. J Cereb Blood Flow Metab 37:2742-2755

8. Ståhl N, Schalén W, Ungerstedt U, Nordström CH (2003) Bedside biochemical monitoring of the penumbra zone surrounding an evacuated acute subdural haematoma. Acta Neurol Scand 108:211-215 